\section{Thyroid nodules with isolated macrocalcification: malignancy risk and diagnostic efficacy of fine-needle aspiration and core needle biopsy}

\author{
Dong Gyu Na', Dae Sik Kim ${ }^{1,2}$, Soo Jin Kim,3, Jae Wook Ryoo ${ }^{4}$, So Lyung Jung ${ }^{5}$ \\ ${ }^{1}$ Department of Radiology, Human Medical Imaging and Intervention Center, Seoul; \\ ${ }^{2}$ Department of Radiology, Incheon Medical Center, Incheon; ${ }^{3}$ Department of Radiology, New \\ Korea Hospital, Gimpo; ${ }^{4}$ Department of Radiology, Samsung Medical Center, Sungkyunkwan \\ University School of Medicine, Seoul; ${ }^{5}$ Department of Radiology, Seoul St. Mary's Hospital, \\ College of Medicine, The Catholic University of Korea, Seoul, Korea
}

Purpose: This study was performed to determine the malignancy risk of thyroid nodules with isolated macrocalcification and to evaluate the diagnostic efficacy of fine-needle aspiration (FNA) and core needle biopsy (CNB).

Methods: From May 2008 to December 2014, a total of 44 patients with isolated macrocalcifications were enrolled from 4,081 consecutive patients who underwent FNA or CNB at a single institution. We assessed the malignancy risk of nodules with isolated macrocalcification. We compared the diagnostic results between FNA and CNB, and the diagnostic efficacy of each procedure was evaluated by the rate of inconclusive results. We compared the diagnostic performance for malignancy between FNA and CNB with a criterion of malignant or atypia/ follicular lesion of undetermined significance (indeterminate) diagnostic results. We investigated whether the ultrasonographic feature of isolated macrocalcification was predictive of malignancy. Results: The malignancy risk of nodules with isolated macrocalcification was $16.1 \%$ in 31 nodules with final diagnoses and $11.4 \%$ in all nodules. CNB demonstrated a significantly lower rate of nondiagnostic and inconclusive results than FNA (7.7\% vs. $53.8 \%, P=0.002$ and $15.4 \%$ vs. $57.7 \%, P=0.003$, respectively) in 26 nodules that underwent both FNA and CNB. CNB showed a marginally higher diagnostic performance for identifying malignancy than FNA $(P=0.067)$. The ultrasonographic features of the anterior margin of isolated macrocalcification were not predictive of malignancy ( $P>0.999)$.

Conclusion: Thyroid nodules with isolated macrocalcification had a low to intermediate malignancy risk and should not be considered benign nodules. CNB showed a higher diagnostic efficacy than FNA in these nodules.

Keywords: Thyroid nodule; Ultrasonography; Diagnosis; Biopsy, fine-needle;

Biopsy, large-core needle

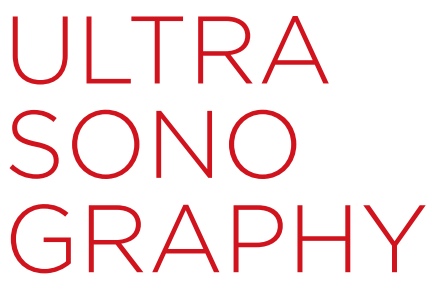

ORIGINAL ARTICLE

http://dx.doi.org/10.14366/usg.15074 pISSN: 2288-5919 - eISSN: 2288-5943 Ultrasonography 2016;35:212-219

Received: November 11, 2015 Revised: December 14, 2015 Accepted: December 27, 2015

Correspondence to: Dong Gyu Na, MD, PhD, Department of Radiology, Human Medical Imaging and Intervention Center, 621 Gangnam-daero, Seocho-gu, Seoul 06524, Korea

Tel. +82-2-512-6695

Fax. $+82-2-512-6646$

E-mail: nndgna@gmail.com

This is an Open Access article distributed under the terms of the Creative Commons Attribution NonCommercial License (http://creativecommons.org/ licenses/by-nc/3.0/) which permits unrestricted noncommercial use, distribution, and reproduction in any medium, provided the original work is properly cited.

Copyright (C) 2016 Korean Society of Ultrasound in Medicine (KSUM)

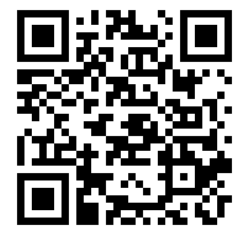

How to cite this article:

Na DG, Kim DS, Kim SJ, Ryoo JW, Jung SL. Thyroid nodules with isolated macrocalcification: malignancy risk and diagnostic efficacy of fine-needle aspiration and core needle biopsy. Ultrasonography. 2016 Jul;35(3):212-219. 


\section{Introduction}

Ultrasonography (US) plays an essential role in estimating the malignancy risk of a thyroid nodule, and the US patterns of thyroid nodules can reliably predict the malignancy risk [1-3]. Although macrocalcifications are associated with a higher malignancy risk in thyroid nodules $[4,5]$, they are not highly specific for malignancy [1$3]$ and have a variable malignancy risk $(23.9 \%-64.8 \%)$ [4-6]. An isolated macrocalcification can be defined as an entirely calcified nodule without any solid component on US [7] and is an uncommon US pattern among thyroid nodules with calcifications [6]. A US lexicon for isolated macrocalcification has not been established, and the isolated macrocalcification has been described as a rim calcification $[8,9]$ or one type of macrocalcification [10].

A previous study [6] reported that malignant tumors were not found in any of 10 nodules with isolated calcification. Although nodules with isolated macrocalcification have been considered benign nodules [7,11], data regarding the malignancy risk of nodules with isolated macrocalcification is insufficient. In our experience, the cytology diagnosis with fine-needle aspiration (FNA) seems less effective in nodules with isolated macrocalcification. A recent study [12] reported that the nondiagnostic rate of FNA was high in thyroid nodules with macrocalcifications, and core needle biopsy (CNB) was more effective for the diagnosis than FNA in these nodules.

The aim of this study was to determine the malignancy risk of thyroid nodules with isolated macrocalcification and to evaluate the diagnostic efficacy of FNA and CNB in these nodules.

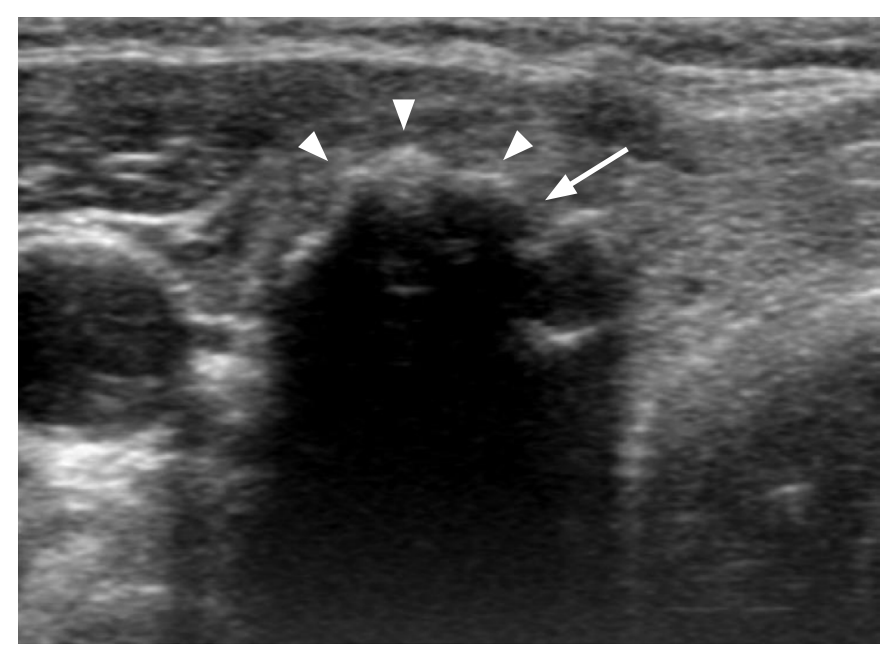

A

\section{Materials and Methods}

The Institutional Review Board approved this retrospective study, and the requirement to obtain informed consent was waived.

\section{Patients}

This study included 44 consecutive patients (38 women, 6 men; mean age, $54 \pm 12$ years) who underwent FNA or CNB for nodules with isolated macrocalcifications. These patients were selected from the 4,081 consecutive patients in whom FNA or CNB was performed for thyroid nodules at a single institution from May 2008 to December 2014. The nodules with isolated macrocalcification were retrospectively identified by reviewing thyroid ultrasounds of 408 patients in which the word "macrocalcification" was mentioned in the radiology report for thyroid US. The nodule with isolated macrocalcification was defined as an entirely calcified nodule with strong posterior acoustic shadowing in which any solid component was not obviously identified within the nodule on US (Figs. 1-3). In two patients with isolated macrocalcifications, neck computed tomography (CT) images were obtained prior to the FNA or CNB procedure.

FNA or CNB was routinely performed for large $(\geq 1 \mathrm{~cm})$ isolated macrocalcifications, and it was selectively performed for small $(<1$ $\mathrm{cm}$ ) isolated macrocalcifications, which mostly included nodules with previous inconclusive FNA results including nondiagnostic or atypia of undetermined significance/follicular lesion of undetermined significance (AUS/FLUS) results at other hospitals, nodules located in the contralateral lobe in candidates for thyroid lobectomy, and

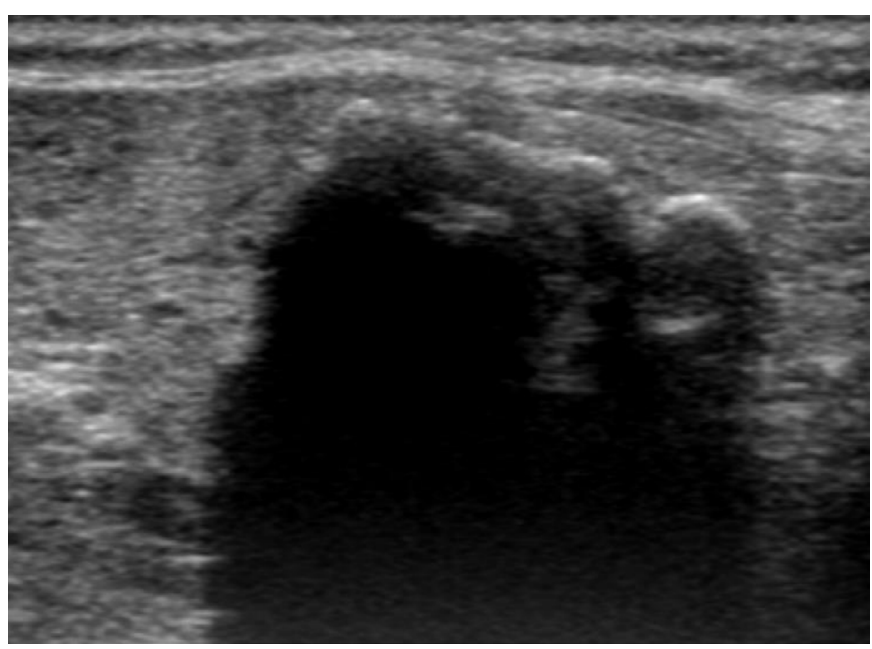

B

Fig. 1. Benign nodule with isolated macrocalcification in a 56-year-old woman.

Transverse sonogram (A) shows a calcified nodule $(18 \mathrm{~mm}$ ) with a lobulated contour (arrowheads) and interruption (arrow) of the anterior margin. The posterior margin of the calcified nodule is not visualized by strong posterior acoustic shadowing on transverse or longitudinal ultrasonography $(A, B)$. 


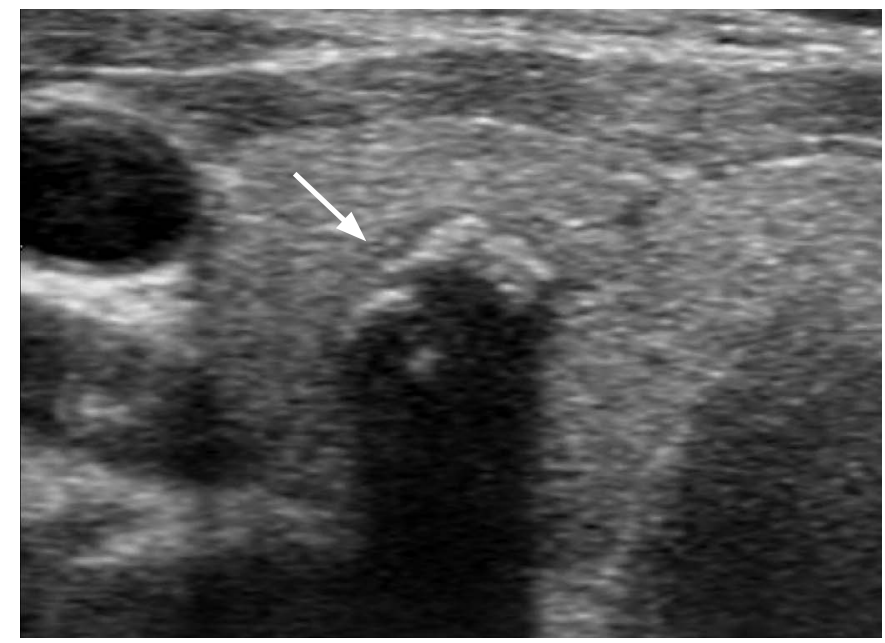

A

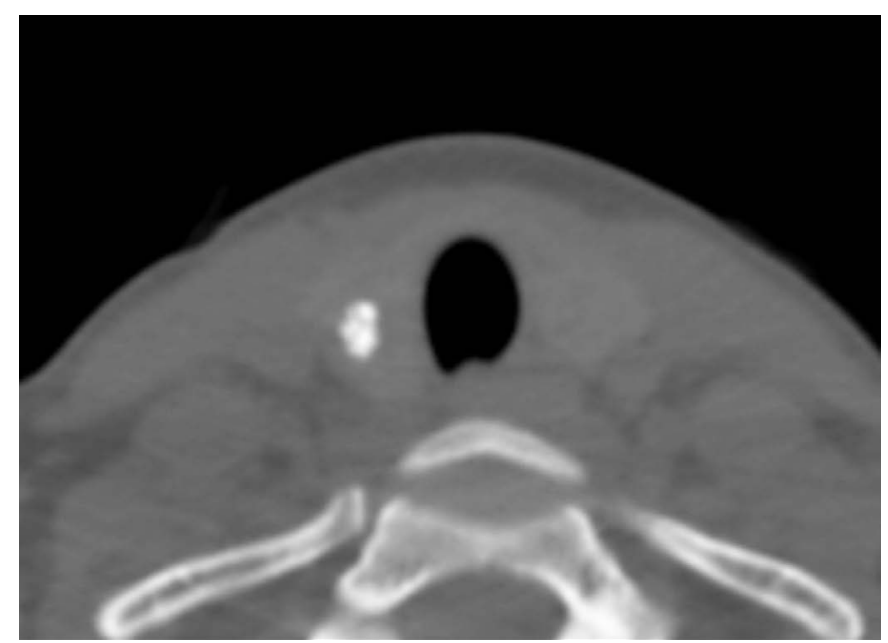

C

nodules for which biopsy was requested by the referring physician. Final diagnoses of malignant tumors were determined by surgery or malignant diagnosis of FNA or CNB, and final diagnoses of benign nodules were determined by benign diagnosis of FNA or CNB. In nodules with AUS/FLUS results at the initial FNA or indeterminate results at the initial CNB, the final diagnosis of benign nodules was determined by benign results of FNA or CNB repeated at least twice.

\section{US Exam and Image Analysis}

High-resolution US scanning using a 10-12 MHz linear-array transducer (AplioXG, Toshiba, Otawarashi, Japan) was employed. Sonograms were retrospectively reviewed by one experienced thyroid radiologist (D.G.N.) who had 19 years of experience in performing thyroid US and interventional procedures. The reviewer, who had no previous knowledge of the FNA result or final diagnosis, determined the presence of isolated macrocalcification from the

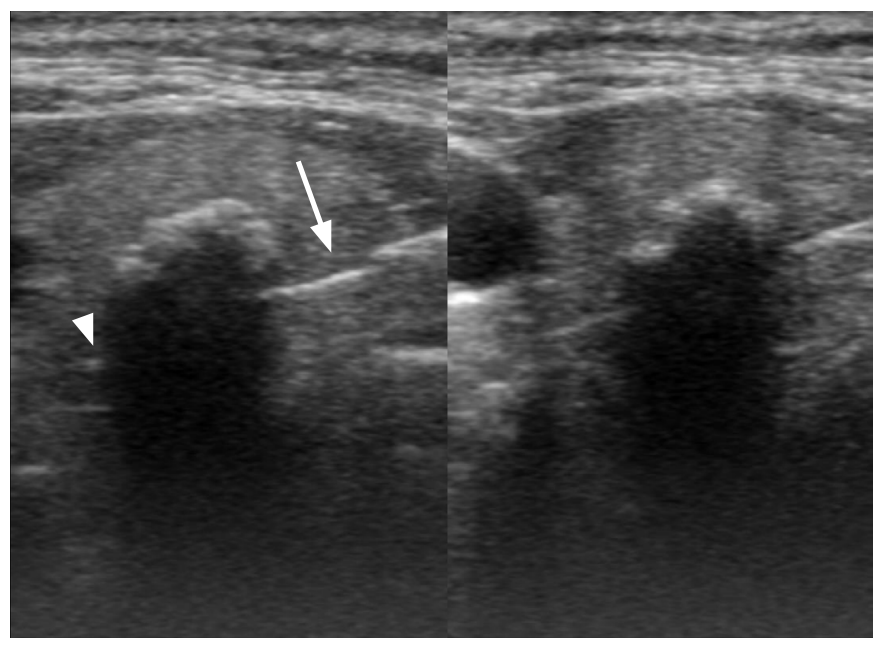

B

Fig. 2. Benign nodule with isolated macrocalcification in a 39-year-old woman.

A. Transverse sonogram shows a calcified nodule $(9 \mathrm{~mm})$ with slightly lobulated contour and interruption (arrow) of the anterior margin. B. The tip (arrowhead) and specimen notch (arrow) of a partially penetrated stylet cannula are seen on the ultrasound obtained immediately after firing of the stylet cannula under the hyperechoic anterior margin through the medial approach (left). An ultrasonography (right) shows the full penetration of the stylet cannula into the nodule by manually advancing the stylet cannula within the nodule. C. Computed tomography demonstrates that the isolated macrocalcification correlates with compact coarse macrocalcification.

database of patients, and retrospectively assessed US features of isolated macrocalcifications at the anterior margin, which included the contour (smooth or lobulated) and the presence of focal interruption of calcification.

\section{US-Guided FNA and CNB Procedures}

US-guided FNA was performed with a conventional method and at least two samples were taken from each nodule [13]. Additional FNA sampling was performed if the aspirated specimen was inadequate by visual assessment. For the FNA, a 23-gauge needle was routinely used, and a 21-gauge needle was selectively used. FNA was performed after the needle tip was positioned inside the calcified nodule by needle penetration through the disrupted portion of calcification, or FNA was performed at the margin of a nodule when the needle penetration was not possible. Direct smears or the liquid-based cytology (LBC) method was used for the preparation 
of FNA specimens. The smears were immediately fixed with alcohol after FNA in the direct smear method, and the specimens were prepared using the ThinPrep 2000 slide preparation device (Hologic Co., Marlborough, MA, USA) in the LBC method. In the LBC method, cytology aspirates in aspiration needles were rinsed immediately in a vial containing $20 \mathrm{~mL}$ of CytoLyt preservative solution. CNB was also performed for the same nodule during the biopsy procedure when aspirated specimens were scanty and inadequate by visual assessment.

CNB was performed using a disposable 18-gauge, double-action spring-activated needle $(1.1 \mathrm{~cm}$ excursion, TSK Acecut, Create Medic, Yokohama, Japan) as described elsewhere $[14,15]$. Using a free-hand technique, the needle tip was manually advanced to the margin or within the nodule, and the stylet and cutting cannula of the needle were fired sequentially. The firing of a cutting cannula was performed after the specimen notch of a stylet cannula was positioned properly inside the nodule. When the needle could not penetrate the center of a calcified nodule, the cutting cannula was fired after the stylet cannula manually penetrated the peripheral portion of a nodule and after positioning the specimen notch facing towards the center of a nodule.

Two CNB samples were obtained at each nodule. All biopsy specimens were placed immediately into 10\% neutral buffered formalin solution and were fixed and stained in the standard fashion for a histological examination. The interpretation of FNA was based on the Bethesda system for reporting thyroid cytopathology [16], and the six categories of a CNB pathology reporting system were used for the interpretation of CNB [17]. After patients underwent biopsy, we immediately compressed the biopsy site, and they were observed with self manual compression of the biopsy site for 20 30 minutes. We made an effort to obtain the qualified cytology and histology specimens by visual assessment of the FNA or CNB specimen at each FNA and CNB procedure.

\section{Data Analysis and Statistics}

We assessed the malignancy risk of nodules with isolated macrocalcification. We compared the diagnostic results of FNA and CNB in nodules with isolated macrocalcification and evaluated the diagnostic efficacy of FNA and CNB by the rate of inconclusive results. We investigated whether US features of isolated macrocalcification at the anterior margin were predictive of malignancy. McNemar's test was used to compare each diagnostic result of FNA and CNB in nodules that underwent both FNA and CNB. We compared the diagnostic performance for malignancy between FNA and CNB with a criterion of a malignant diagnostic result and with a criterion of a malignant or AUS/FLUS (indeterminate) diagnostic result by receiver operating characteristic (ROC) analysis. The chi-square or Fisher exact test was used for determining the association of US features and malignancy. Statistical analysis was performed with the IBM SPSS Statistics for Windows software package ver. 19.0 (IBM Co., Armonk, NY, USA), and a significant difference was defined as a P-value less than 0.05 .

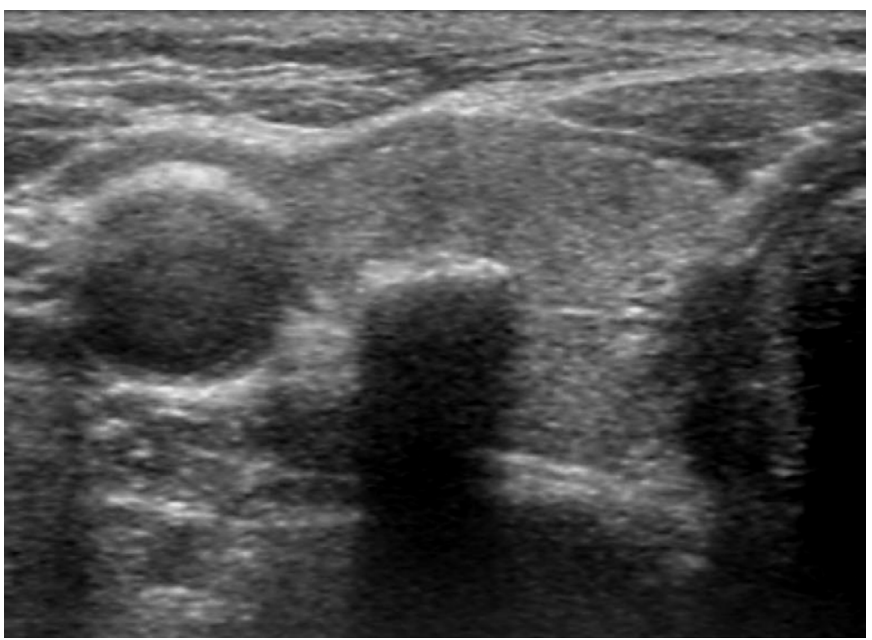

A

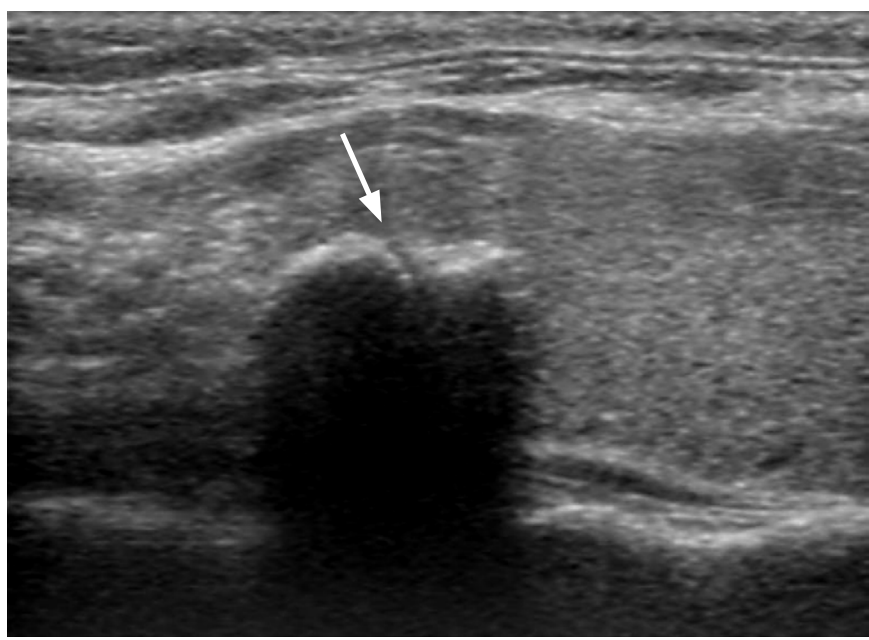

B

Fig. 3. Papillary carcinoma manifested as an isolated macrocalcification in a 56-year-old woman.

A. Transverse sonogram shows a calcified nodule $(18 \mathrm{~mm})$ with a smooth contour of the anterior margin. B. Longitudinal sonogram shows focal interruption (arrow) of the anterior margin of the calcified nodule. Suspicious metastatic lymph nodes were detected in the ipsilateral lateral neck by ultrasonography (not shown), and metastatic lymph nodes and minimal extrathyroidal extension of the tumor were found by surgery. 


\section{Results}

\section{Demographic Data}

Isolated macrocalcification was found in 44 of 4,081 patients $(1.1 \%)$ who underwent FNA or CNB. The size of the nodules ranged from 4 to $18 \mathrm{~mm}$ (mean size, $8.6 \pm 3.6 \mathrm{~mm}$ ). Among 44 nodules that underwent FNA or CNB, both FNA and CNB were performed simultaneously in 26 nodules (59.1\%), FNA alone in $13(29.5 \%)$ and CNB alone in five nodules (11.4\%) for the initial biopsy procedure. First and second repeat FNA were performed in seven and two nodules, and first and second repeat CNB were performed in five and two nodules, respectively. Among the five patients with malignant tumors, clinically apparent lymph node (LN) metastasis was detected by preoperative US in two patients. In three patients who had malignant tumors and underwent thyroid surgery, LN metastasis was found in all patients; among them, LN metastasis was found only in the central neck $(n=1)$, in both the central and lateral neck $(n=1)$, and in only the lateral neck $(n=1)$. Minimal extrathyroidal extension of a malignant tumor was found in two of the three patients who underwent surgery. Clinically apparent LN metastasis or extrathyroidal tumor extension was found only in two papillary carcinomas in which the maximal tumor size was $12 \mathrm{~mm}$ in one and $19 \mathrm{~mm}$ in the other. There were no major complications such as serious hemorrhage in any of the patients after FNA or CNB, and none required hospital admission or intervention.

\section{Malignancy Risk of Nodules with Isolated Macrocalcification} Final diagnoses were obtained in 31 of 44 nodules $(70.4 \%)$, which included 26 benign nodules (83.9\%), and five malignant nodules $(16.1 \%)$. Final diagnoses of benign nodules were determined by one benign result of FNA or CNB in 13 nodules and by two benign results in 13 nodules. Final diagnoses of the five cases with malignant tumors were diagnosed by surgical resection in three patients (all conventional papillary carcinomas) and by a malignant diagnosis upon FNA or CNB in two nodules. The calculated malignancy rate of nodules with isolated macrocalcification was $16.1 \%$ in 31 nodules with final diagnoses with the abovementioned reference of final diagnosis and $11.4 \%$ among all nodules. In 31 nodules with final diagnoses, there was no significant difference in the malignancy risk between small $(<1 \mathrm{~cm}, \mathrm{n}=19)$ and large $(\geq 1$ $\mathrm{cm}, \mathrm{n}=12$ ) isolated macrocalcifications ( $15.8 \%$ vs. $16.7 \%, \mathrm{P}>0.999$, respectively).

\section{Diagnostic Efficacy of FNA and CNB}

Table 1 demonstrates the diagnostic results of FNA and CNB in nodules with isolated macrocalcification. A nondiagnostic result of FNA and CNB was found in $61.5 \%$ and $6.5 \%$, respectively, and inconclusive results including nondiagnostic or AUS/FLUS results were found in $66.7 \%$ and $16.1 \%$ of the nodules, respectively. In the 26 nodules that underwent both FNA and CNB, CNB demonstrated a significantly lower rate of nondiagnostic and inconclusive results than FNA ( $7.7 \%$ vs. $53.8 \%, P=0.002$ and $15.4 \%$ vs. $57.7 \%$, $\mathrm{P}=0.003$, respectively). CNB demonstrated a significantly higher rate

Table 1. Diagnostic results of FNA and CNB in isolated macrocalcifications

\begin{tabular}{|c|c|c|}
\hline Diagnosis $^{\mathrm{a})}$ & FNA $(n=39)$ & CNB $(n=31)$ \\
\hline Nondiagnostic & $24(61.5)$ & $2(6.5)$ \\
\hline Benign & $11(28.2)$ & $23(74.2)$ \\
\hline AUS/FLUS ${ }^{b)}$, indeterminate ${ }^{c)}$ & $2(5.1)$ & $3(9.7)$ \\
\hline FN/SFN & 0 & 0 \\
\hline Suspicious for malignancy & 0 & 0 \\
\hline Malignant & $2(5.1)$ & $3(9.7)$ \\
\hline
\end{tabular}

Values are presented as number (\%).

FNA, fine-needle aspiration; CNB, core needle biopsy; AUS/FLUS, atypia of undetermined significance/follicular lesion of undetermined significance; FN/SFN, follicular neoplasm/suspicious for a follicular neoplasm.

a)Diagnosis based on six categories of the Bethesda System for Reporting Thyroid Cytology for FNA and diagnosis based on six categories of a CNB pathology reporting system for CNB. ${ }^{\text {b) }}$ Category for FNA cytology diagnosis. ${ }^{\text {c }}$ Category for CNB histology diagnosis.

Table 2. Diagnostic results of FNA in isolated macrocalcifications with final diagnoses $(n=27)$

\begin{tabular}{lcccc}
\hline \multicolumn{1}{c}{ Diagnosis } & FNA & Benign nodule $(\mathrm{n}=22)$ & Malignant nodule $(\mathrm{n}=5)$ & Malignancy risk $(\%)$ \\
\hline Nondiagnostic & $13(48.1)$ & $10(45.5)$ & $3(60)$ & 0 \\
Benign & $11(40.7)$ & $11(50)$ & 0 & 0 \\
AUS/FLUS & $1(3.7)$ & $1(4.5)$ & - & 0 \\
FN/SFN & 0 & - & - & - \\
Suspicious for malignancy & 0 & - & $2(40)$ & 100 \\
Malignant & $2(7.4)$ & 0 & & - \\
\hline
\end{tabular}

Values are presented as number (\%) unless otherwise indicated.

FNA, fine-needle aspiration; AUS/FLUS, atypia of undetermined significance/follicular lesion of undetermined significance; FN/SFN, follicular neoplasm/suspicious for a follicular neoplasm. 
of benign diagnosis than FNA ( $73.1 \%$ vs. $34.6 \%, P=0.006)$, and there was no difference in the other diagnostic results $(P>0.999)$. A nondiagnostic result at both FNA and CNB was found in only one of the 26 patients (3.8\%) who underwent both FNA and CNB.

In the 31 patients with final diagnoses, FNA was performed in 27 patients and CNB in 29 patients (Tables 2, 3). Malignancy was found in three of 13 nodules (23.1\%) with nondiagnostic results at the initial FNA, and no malignancy was found in one nodule with a nondiagnostic result at the initial CNB. In one nodule that underwent both FNA and CNB, the diagnosis was nondiagnostic and benign at the initial and repeat FNA, respectively; however, the diagnosis of this nodule was indeterminate and malignant at the initial and repeat $C N B$, and finally proved to be a papillary carcinoma by surgery. In one nodule in which the initial CNB diagnosis was benign and the FNA result was nondiagnostic, metastatic LN was diagnosed by FNA and the nodule was proven to be a papillary carcinoma by surgery. One nodule with an AUS/ FLUS diagnosis at the initial FNA was finally diagnosed as a benign nodule by benign results of CNB repeated twice. In two nodules with indeterminate results at the initial CNB, one nodule was finally diagnosed as a benign nodule by benign results of CNB repeated twice, and another nodule was diagnosed as malignant upon repeat CNB and was proven to be a papillary carcinoma by surgery.

In the 25 nodules that underwent both FNA and CNB and were finally diagnosed, CNB showed a higher sensitivity for malignancy than FNA with a criterion of malignant diagnosis (60\% vs. 40\%) and with criteria of malignant and AUS/FLUS or indeterminate diagnosis ( $80 \%$ vs. $40 \%$ ). CNB showed a marginally higher diagnostic performance than FNA $(P=0.067)$ with a criterion of malignant or AUS/FLUS (indeterminate) diagnostic results by

Table 3. Diagnostic results of CNB in isolated macrocalcifications with final diagnoses $(n=29)$

\begin{tabular}{lcccc}
\hline \multicolumn{1}{c}{ Diagnosis } & CNB & Benign nodule $(\mathrm{n}=24)$ & Malignant nodule $(\mathrm{n}=5)$ & Malignancy risk $(\%)$ \\
\hline Nondiagnostic & $1(3.4)$ & $1(4.2)$ & 0 & 0 \\
Benign & $23(79.3)$ & $22(91.7)$ & $1(20)$ & 4.3 \\
Indeterminate & $2(6.9)$ & $1(4.2)$ & 0 & 50 \\
FN/SFN & 0 & - & 0 & - \\
Suspicious for malignancy & 0 & 0 & $3(60)$ & 100 \\
Malignant & $3(10.3)$ & - & 0 \\
\hline
\end{tabular}

Values are presented as number (\%) unless otherwise indicated.

CNB, core needle biopsy; FN/SFN, follicular neoplasm/suspicious for a follicular neoplasm.

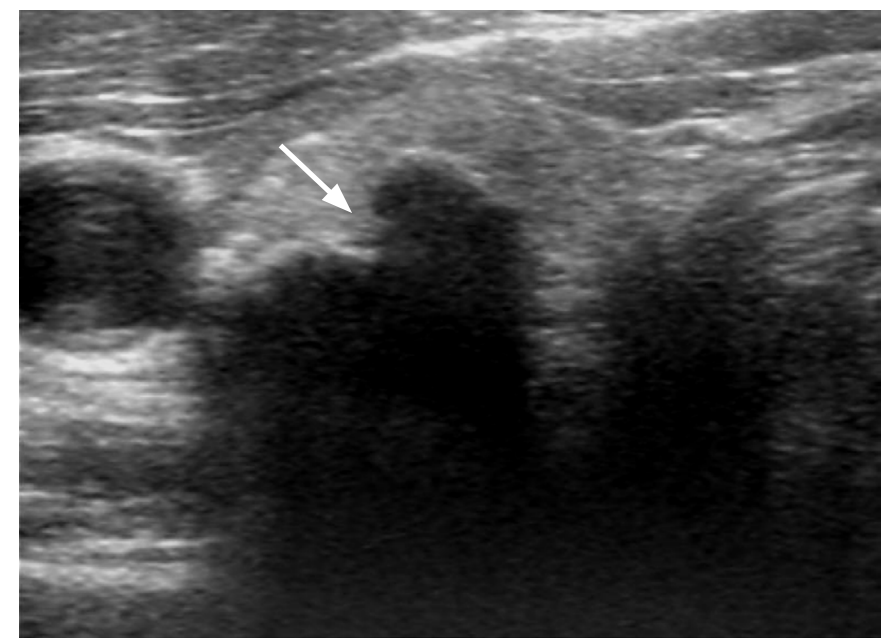

A

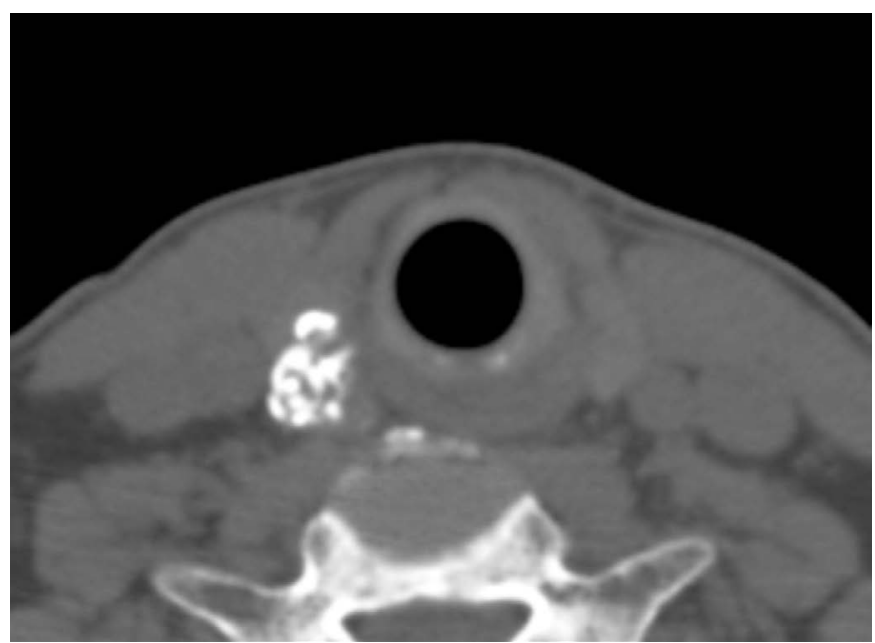

B

Fig. 4. Papillary carcinoma manifested as an isolated macrocalcification in a 62-year-old man.

A. Transverse sonogram shows a calcified nodule $(16 \mathrm{~mm})$ with lobulated contour and focal interruption (arrow) of the anterior margin. B. A computed tomography image demonstrates that the isolated macrocalcification correlates with conglomerated coarse calcifications. Suspicious metastatic lymph nodes were detected in the ipsilateral lateral and central neck by ultrasonography (not shown), and metastatic lymph nodes and extrathyroidal extension of the tumor were found by surgery. 
ROC analysis; however, there was no significant difference in the diagnostic performance between FNA and CNB with a criterion of malignant diagnostic results only $(\mathrm{P}=0.532)$.

\section{US Features of Isolated Macrocalcification}

The US feature of a lobulated contour of the anterior margin was found in three of the five malignant nodules $(60 \%)$ and in 18 of the 26 benign nodules (69.2\%) ( $P>0.999$ ). Focal disruption of calcification at the anterior margin was found in three of the five malignant nodules $(60 \%)$ and in 16 of the 26 benign nodules (61.5\%) ( $P>0.999)$. The US features of lobulated contour and disruption of calcification were found in two of the five malignant nodules (40\%) and in 13 of the 26 benign nodules ( $50 \%)(P>0.999)$ (Figs. 1-4). In the two patients who underwent neck $C T$, the nodules with isolated macrocalcification correlated with coarse calcified nodules on CT images (Figs. 2, 4).

\section{Discussion}

Our data demonstrated that thyroid nodules with isolated macrocalcification had a low to intermediate malignancy risk, with a range of $11.4 \%$ to $16.1 \%$. CNB showed a lower rate of nondiagnostic or inconclusive results than FNA. The US feature of isolated macrocalcification at the anterior margin was not predictive of malignancy.

Our results suggest that thyroid nodules with isolated macrocalcifications detected on US should not be considered benign nodules, and the malignant tumor manifesting as an isolated macrocalcification has the potential for behaving as an aggressive tumor such as clinically apparent $L N$ metastasis or extrathyroidal extension. It may be reasonable to perform a biopsy procedure for large $(\geq 1 \mathrm{~cm})$ nodules with isolated macrocalcification because the malignancy risk of isolated macrocalcification was low to intermediate and clinically apparent $L N$ metastasis or extrathyroidal extension was found only in large $(\geq 1 \mathrm{~cm})$ malignant nodules with isolated macrocalcifications.

A recent study [12] reported that CNB decreased inconclusive diagnoses and increased sensitivity for malignancy compared with FNA in nodules with macrocalcifications. Our study also suggests that CNB could effectively reduce nondiagnostic or inconclusive results in nodules with isolated macrocalcification compared with FNA. Therefore, CNB may be helpful for reducing repeated FNA procedures and diagnostic operations. However, potential for falsenegative results of CNB should not be neglected, considering the false-negative rate (4.3\%) of benign diagnosis on CNB in isolated macrocalcifications, which seems slightly higher than the overall false-negative rate of CNB in thyroid nodules [14].

The CNB procedure may be somewhat difficult in nodules with isolated macrocalcification, and several technical factors should be taken into consideration for a successful CNB procedure. First, we used a powerful spring-activated double-action CNB device for effective needle penetration. The spring-activated firing of a stylet cannula at the site of interrupted calcification under the anterior hyperechoic margin by a medial approach was useful for the effective penetration of the needle into the center of a calcified nodule. In the case of limited partial penetration of a stylet cannula into the calcified nodule, additional manual needle penetration is necessary by carefully advancing the stylet needle into the nodule (Fig. 2B). Second, when the needle penetration into the center of an isolated macrocalcification is not possible by spring-activated firing due to compact calcifications, the manual penetration of a stylet cannula into the calcified nodule can be performed at the site of interrupted calcification by slowly advancing the stylet cannula using a controlled screwing action into the nodule.

US has a limitation in the assessment of nodules with isolated macrocalcification because the internal content and posterior margin of the nodule cannot be visualized due to strong posterior acoustic shadowing. Therefore, the US feature of isolated macrocalcification cannot exclude the presence of a solid component hidden by posterior acoustic shadowing on US. CT images obtained from two patients suggest that the US feature of isolated macrocalcification correlates with dense coarse calcifications and that an isolated macrocalcification should be differentiated from a rim or eggshell calcification. In large nodules with isolated macrocalcifications, enhanced neck CT may provide information about the actual extent and characteristics of calcifications and possibly any hidden solid portion, which might be helpful for determining the target site for biopsy.

There are several limitations to this study. First, the retrospective assessment of ultrasounds by an interpreter has an inevitable limitation in the interpretation of isolated macrocalcifications. Second, there exists a selection bias for small $(<1 \mathrm{~cm})$ isolated macrocalcifications because FNA or CNB was not routinely performed for all small isolated macrocalcifications. This might have affected the malignancy risk calculated for small nodules with isolated macrocalcifications. Third, the possible false-negative result of CNB due to technical difficulty in penetrating the calcified nodule might have affected the results of our study. Fourth, CT images were not obtained in the majority of patients, and we could not determine the $\mathrm{CT}$ characteristics of isolated macrocalcifications.

In conclusion, thyroid nodules with isolated macrocalcifications have a low to intermediate malignancy risk $(11.4 \%-16.1 \%)$ and should not be considered benign nodules. CNB showed a higher diagnostic efficacy than FNA in these nodules. 
ORCID: Dong Gyu Na: http://orcid.org/0000-0001-6422-1652; Dae Sik Kim: http:// orcid.org/0000-0001-8424-8561; Soo Jin Kim: http://orcid.org/0000-0001-82331388; Jae Wook Ryoo: http://orcid.org/0000-0003-4695-6246; So Lyung Jung: http:// orcid.org/0000-0002-3267-8399

\section{Conflict of Interest}

No potential conflict of interest relevant to this article was reported.

\section{Acknowledgments}

This study was supported in part by the Research Fund of the Korean Society of Ultrasound in Medicine.

\section{References}

1. Brito JP, Gionfriddo $M R, A l$ Nofal $A$, Boehmer $K R$, Leppin $A L$, Reading $C$, et al. The accuracy of thyroid nodule ultrasound to predict thyroid cancer: systematic review and meta-analysis. J Clin Endocrinol Metab 2014;99:1253-1263.

2. Campanella P, Ianni F, Rota CA, Corsello SM, Pontecorvi A. Quantification of cancer risk of each clinical and ultrasonographic suspicious feature of thyroid nodules: a systematic review and meta-analysis. Eur J Endocrinol 2014;170:R203-R211.

3. Remonti LR, Kramer CK, Leitao CB, Pinto LC, Gross JL. Thyroid ultrasound features and risk of carcinoma: a systematic review and meta-analysis of observational studies. Thyroid 2015;25:538-550.

4. Moon WJ, Jung SL, Lee JH, Na DG, Baek JH, Lee YH, et al. Benign and malignant thyroid nodules: US differentiation: multicenter retrospective study. Radiology 2008;247:762-770.

5. Seo H, Na DG, Kim JH, Kim KW, Yoon JW. Ultrasound-based risk stratification for malignancy in thyroid nodules: a four-tier categorization system. Eur Radiol 2015;25:2153-2162.

6. Lu Z, Mu Y, Zhu H, Luo Y, Kong Q, Dou J, et al. Clinical value of using ultrasound to assess calcification patterns in thyroid nodules. World J Surg 2011;35:122-127.

7. Russ G. Risk stratification of thyroid nodules on ultrasonography with the French TI-RADS: description and reflections. Ultrasonogra- phy 2016:35:25-38.

8. Yoon DY, Lee JW, Chang SK, Choi CS, Yun EJ, Seo YL, et al. Peripheral calcification in thyroid nodules: ultrasonographic features and prediction of malignancy. J Ultrasound Med 2007;26:13491355.

9. Kim BM, Kim MJ, Kim EK, Kwak JY, Hong SW, Son EJ, et al. Sonographic differentiation of thyroid nodules with eggshell calcifications. J Ultrasound Med 2008;27:1425-1430.

10. Park YJ, Kim JA, Son EJ, Youk JH, Kim EK, Kwak JY, et al. Thyroid nodules with macrocalcification: sonographic findings predictive of malignancy. Yonsei Med J 2014;55:339-344.

11. Russ $G$, Royer B, Bigorgne $C$, Rouxel $A$, Bienvenu-Perrard $M$, Leenhardt $\mathrm{L}$. Prospective evaluation of thyroid imaging reporting and data system on 4550 nodules with and without elastography. Eur J Endocrinol 2013;168:649-655.

12. Yi KS, Kim JH, Na DG, Seo H, Min HS, Won JK, et al. Usefulness of core needle biopsy for thyroid nodules with macrocalcifications: comparison with fine-needle aspiration. Thyroid 2015;25:657-664.

13. Lee YH, Baek JH, Jung SL, Kwak JY, Kim JH, Shin JH, et al. Ultrasoundguided fine needle aspiration of thyroid nodules: a consensus statement by the korean society of thyroid radiology. Korean J Radiol 2015;16:391-401.

14. Sung JY, Na DG, Kim KS, Yoo H, Lee H, Kim JH, et al. Diagnostic accuracy of fine-needle aspiration versus core-needle biopsy for the diagnosis of thyroid malignancy in a clinical cohort. Eur Radiol 2012;22:1564-1572.

15. Na DG, Min HS, Lee H, Won JK, Seo HB, Kim JH. Role of core needle biopsy in the management of atypia/follicular lesion of undetermined significance thyroid nodules: comparison with repeat fine-needle aspiration in subcategory nodules. Eur Thyroid J 2015;4:189-196.

16. Cibas ES, Ali SZ. The Bethesda System for Reporting Thyroid Cytopathology. Thyroid 2009;19:1159-1165.

17. Jung CK, Min HS, Park HJ, Song DE, Kim JH, Park SY, et al. Pathology reporting of thyroid core needle biopsy: a proposal of the Korean Endocrine Pathology Thyroid Core Needle Biopsy Study Group. J Pathol Transl Med 2015;49:288-299. 